\title{
Induction and impairment of saturated yaw and surge vection
}

\author{
F. A. M. VAN DER STEEN and P. T. M. BROCKHOFF \\ Delft University of Technology, Delft, The Netherlands
}

\begin{abstract}
A flight simulator was used to investigate the perception of self-motion and visual scene motion during the induction of saturated $10 \mathrm{deg} / \mathrm{sec}$ yaw and $50 \mathrm{~m} / \mathrm{sec}$ surge vection, and during subsequent impairment of saturated vection by inertial motions. The subjects $(n=5)$ did not perceive any selfacceleration or visual scene deceleration during the induction of saturated vection but perceived a rather sudden change in self-velocity and visual scene velocity. The mean group times to saturated vection were $3.0 \mathrm{sec}$ for yaw and $2.7 \mathrm{sec}$ for surge. Above certain inertial motion amplitudes, the subjects reported additional self-motion from the applied inertial motions while experiencing saturated vection. To impair saturated yaw vection, these amplitudes were $0.6 \mathrm{~m} / \mathrm{sec}^{2}, 0.4 \mathrm{~m} / \mathrm{sec}^{2}, 8 \mathrm{deg} / \mathrm{sec}^{2}$, and $5 \mathrm{deg} / \mathrm{sec}^{2}$, for surge, sway, roll and yaw motions, respectively. To impair saturated surge vection, these amplitudes were $0.6 \mathrm{~m} / \mathrm{sec}^{2}, 0.3 \mathrm{~m} / \mathrm{sec}^{2}, 5 \mathrm{deg} / \mathrm{sec}^{2}$, and $4 \mathrm{deg} / \mathrm{sec}^{2}$, respectively. The results indicate that saturated vection is more robust for translations than for rotations because the rotational inertial amplitudes were closer to the amplitudes at which the applied inertial motion was perceived than the translational inertial amplitudes.
\end{abstract}

When one is sitting in a train and an adjacent train starts to move, one may perceive self-motion while the adjacent train is perceived as stationary. This train illusion is the perhaps best known of many examples demonstrating that a scene that moves with respect to the earth's surface can induce perceived self-motion in an earthstationary observer. This kind of self-motion perception is usually referred to as vection (Fischer \& Kornmüller, 1930). Vision is the best documented modality that induces vection (Berthoz \& Droulez, 1982; Berthoz, Pavard, \& Young, 1975; Brandt, Wist, \& Dichgans, 1971; Dichgans \& Brandt, 1978; Howard, 1982, 1986; Mach, $1875 / 1967)$. Vection can, however, be elicited by other sensory modalities that register the environmental scene. Auditive (Lackner, 1977), somatosensory (Lackner \& DiZio, 1984), or combined somatosensory and kinaesthetic (Bles, 1981; Brandt, Büchele, \& Arnold, 1977) information about the environment has been shown to induce vection. In relation to vection in the context of this article, however, vision is considered to be the arousing sensory modality. Vection is called "saturated" when visual scene motion has induced self-motion through that scene at the motion amplitude of the visual scene while the visual scene is perceived as earth stationary (Wertheim, 1994). As a result, the amplitude of perceived self-motion

The Netherlands National Aerospace Laboratory (NLR) is kindly acknowledged for providing access to the NSF setup. This research was sponsored by a grant from the Royal Netherlands Airforce (RNLAF) and by the Netherlands Organisation for Scientific Research (NWO). Correspondence should be addressed to F. A. M. van der Steen, Siemens-The Netherlands, P. O. Box 16068, NL-2600 BB The Hague, The Netherlands (e-mail: han.steen-van-der(@siemens.nl). during saturated vection can easily be resolved by determining the perception of an earth-stationary visual scene.'

The dynamics of the induction of saturated vection have been investigated using different kinds of experimental setups such as rotating chairs, sleds, and flight simulators, and providing visual stimuli that cover specific parts of the visual field (e.g., Andersen \& Braunstein, 1985; Brandt, Dichgans, \& Koenig, 1973) or the entire visual field (e.g., Büttner \& Henn, 1981; Dichgans \& Brandt, 1978). It was demonstrated earlier (e.g., Berthoz \& Droulez, 1982) that saturated vection can be induced when the visual scene suggests translatory self-motion at a constant velocity in an inertial reference frame - that is, self-motion that is free from linear and angular accelerations of the body. The only rotational self-motion in which no significant forces or angular accelerations occur is a (not a too high) constant angular velocity about the gravitational vertical, provided that the observer does not make any head or limb movements that would result in centrifugal and Coriolis accelerations. Research to determine the dynamics of saturated vection mainly has concentrated on this kind of self-motion using rotating chairs and visual displays covering a large portion of the visual field and having unlimited angular displacement (Büttner \& Henn, 1981; Dichgans \& Brandt, 1978; Howard, 1982). Experiments using vertically striped "optokinetic drums," for example, showed that earth-stationary observers perceive a gradual buildup of self-rotation velocity when exposed to a step in visual yaw velocity from zero to a certain amplitude (Brandt et al., 1973; Dichgans \& Brandt, 1978) while perceived drum velocity with respect to the laboratory gradually decreased. Eventually, saturated vection was induced after about $10 \mathrm{sec}$, as evidenced by the perception of self-rotation inside a laboratory- 
stationary perceived drum that was, up to a certain visual angular velocity (Brandt et al., 1973), indistinguishable from actual self-rotation in the laboratory (Brandt et al., 1971). Similar results were obtained from experiments in which visual scene motion induced a translatory forward perception of self-motion (see Dichgans \& Brandt, 1978, and Berthoz \& Droulez, 1982, for reviews). Apparently, a change in perceived self-velocity and a concurrent change in perceived visual scene velocity had occurred in between the instants of exposure to visual scene motion and the presence of saturated vection. This leads to the first question addressed in the present experiments: Does an observer perceive any acceleration of the body and of the visual scene while saturated vection is induced by a step in visual scene velocity? Despite reports about "dropouts" or "waxing and waning" of vection (e.g., Dichgans \& Brandt, 1978), this question has not yet been quantitatively answered. The second question addresses the robustness of the induced saturated vection to inertial motions: What amplitude of an inertial motion profile is needed to impair saturated vection as evidenced by a visual scene that is no longer perceived as earth stationary? This robustness can be used to predict the maximum amount of inertial stimulation that can be applied while the visual scene is still perceived as earth stationary and, hence, perceived self-motion through a visual scene is perceived as realistic (van der Steen, 1998).

Two types of visual scene motion were tested that are known to elicit saturated vection: yaw motion about the vertical axis and translatory forward motion (surge). Saturated vection was induced by presenting a moving visual scene representing a natural landscape, in a flight simulator that was equipped with a wide-visual system and a 6-dof motion platform system. First, the time to saturation was measured following a step in visual scene velocity. The robustness of saturated vection was subsequently tested by providing inertial motion stimuli while the visual motion with respect to the subject remained constant. The inertial motions had an accelerationdeceleration profile that regularly occurred both during natural body movements and during body motions in a vehicle. The inertial motion amplitudes at which saturated vection was impaired were determined both for rotations: roll (rotation about the longitudinal body axis) and yaw (rotation about the vertical axis), and for translations: surge (forward-backward motion) and sway (lateral side-to-side motion). In addition, the subjects were instructed to report perceived self-motion and visual scene motion throughout the experimental sessions.

\section{APPARATUS}

The experiments were conducted using the National Simulation Facility (NSF) at the Netherlands National Aerospace Laboratory (NLR) in Amsterdam (Figure 1). The NSF consists of an F-16 cockpit (General Dynamics) surrounded by a wide-visual dome display (Evans
\& Sutherland) that is mounted on a 6-dof motion base (Hydraudyne).

\section{Subject Position}

The subject was seated in the cockpit chair. This chair had a backward tilt of about $30 \mathrm{deg}$ but the headrest was about vertical. The cockpit chair was adjusted in height for each subject in order to keep the center of each subject's eyes (the eye reference point) at the same location. The subject was strapped in the seat but the head and limbs were free to move.

\section{Response and Communication}

A side-stick (a device for manual flight control consisting of a rigid stick that pivots on two axes) with several push buttons was positioned near the subject's right hand. The side-stick and one of the push buttons were used to register the subject's responses. The subject wore a helmet with two earphones and a microphone for communication with the experimenter. In addition, the sound of the simulator base was masked by providing $90-\mathrm{dB}$ white noise through the ear phones.

\section{Visual Display}

The projected (full color) visual scene (VistaView) inside the dome was nearly rectangular and extended 142 $\times 110 \mathrm{deg}$ (horizontal $\times$ vertical; Figure 2 ).

The scene was a computer-generated image (Silicon Graphics Challenge L) representing a natural landscape. The display resolution was 21.2 arcmin per optical line pair (OLP). ${ }^{2}$ The average luminance was $4.2 \mathrm{ftL}$. An elliptic high-resolution inset in the center of the display extended $52 \times 36 \mathrm{deg}$ (Figure 2). The inset resolution was $7.1 \mathrm{arcmin} / \mathrm{OLP}$. The luminance was $6.8 \mathrm{ftL}$ in the inset center, and $6.6 \mathrm{ftL}$ on average. The image update and refresh rate were $30 \mathrm{~Hz}$. The subject looked at the visual scene through a head-up display with negligible light absorption. The lower midpart of the scene was occluded by the cockpit front (Figure 3).

\section{METHOD}

Yaw and surge vection were tested in separate experiments. Although the visual scene layout and motion were different for yaw and surge, the method used was equal in both experiments.

\section{Subjects}

Five healthy male students, age 23 to 31 , voluntarily participated as subjects in the experiments. One of the authors also participated and was the only non-naive subject. None of the subjects reported having a history of extreme sensitivity to motion sickness or vertigo. One of the (naive) subjects had corrected vision ( -2 diopters). Each subject reported having perceived an earth-stationary visual scene during self-motion in daily life.

\section{Yaw Vection Visual Stimulus}

Figure 4 depicts a part of the visual scene used in the yaw vection experiment. The scene represented a natural landscape and included a large number of objects with vertical polarity, such as 


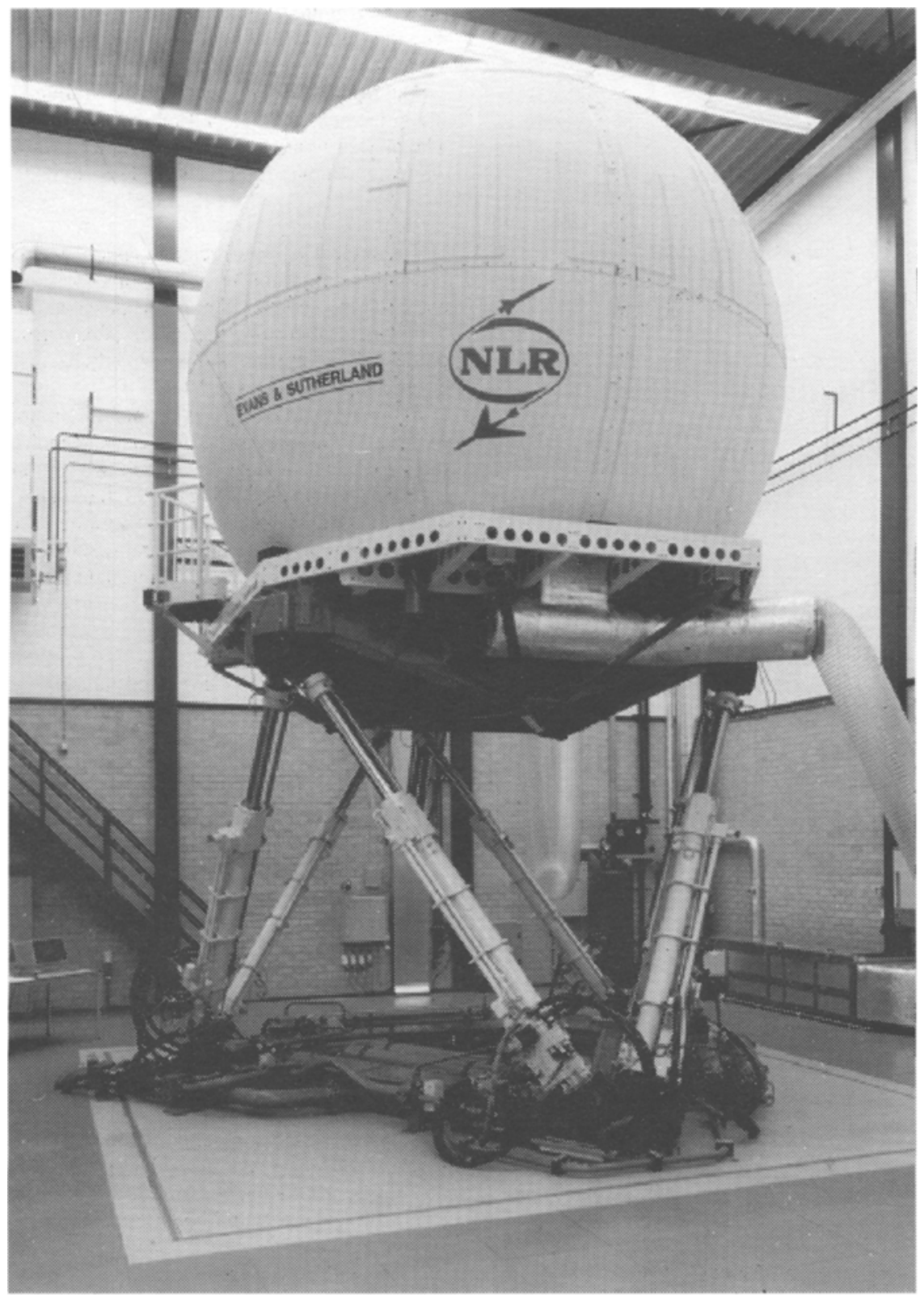

Figure 1. The National Simulation Facility at the National Aerospace Laboratory in Amsterdam in 1995, which was used in the experiments. Reprinted from Journal of Vestibular Research, 8, F. A. M. van der Steen, An earth-stationary perceived visual scene during roll and yaw motions in a flight simulator, 411-425, Copyright 1998, with permission from Elsevier Science.

trees, in order to provide abundant visual information for yaw motion. Five meters was selected as the viewpoint height above the terrain in order to expose the horizon line in the vertically central part of the high-resolution inset.

\section{Surge Vection Visual Stimulus}

Figure 5 depicts subsequent parts of the visual scene used in the surge vection experiment. The viewpoint height above visual scene terrain was $50 \mathrm{~m}$. The low viewpoint height was chosen to expose abundant visual motion information for surge through a high optic flow magnitude. Because of the gently wavy landscape tested, however, a very low height was avoided in order to avoid large fluctuations in height and therefore in optic flow magnitude. The maximum variation in height above terrain was approximately $5 \mathrm{~m}$.

\section{Visual Motion}

Each experimental session started with a visual scene that was stationary with respect to the subject for $2 \mathrm{sec}$. Subsequently, the visual scene was set at a constant velocity with respect to the subject. This velocity was $10 \mathrm{deg} / \mathrm{sec}$ leftward for yaw and $50 \mathrm{~m} / \mathrm{sec}$ toward 

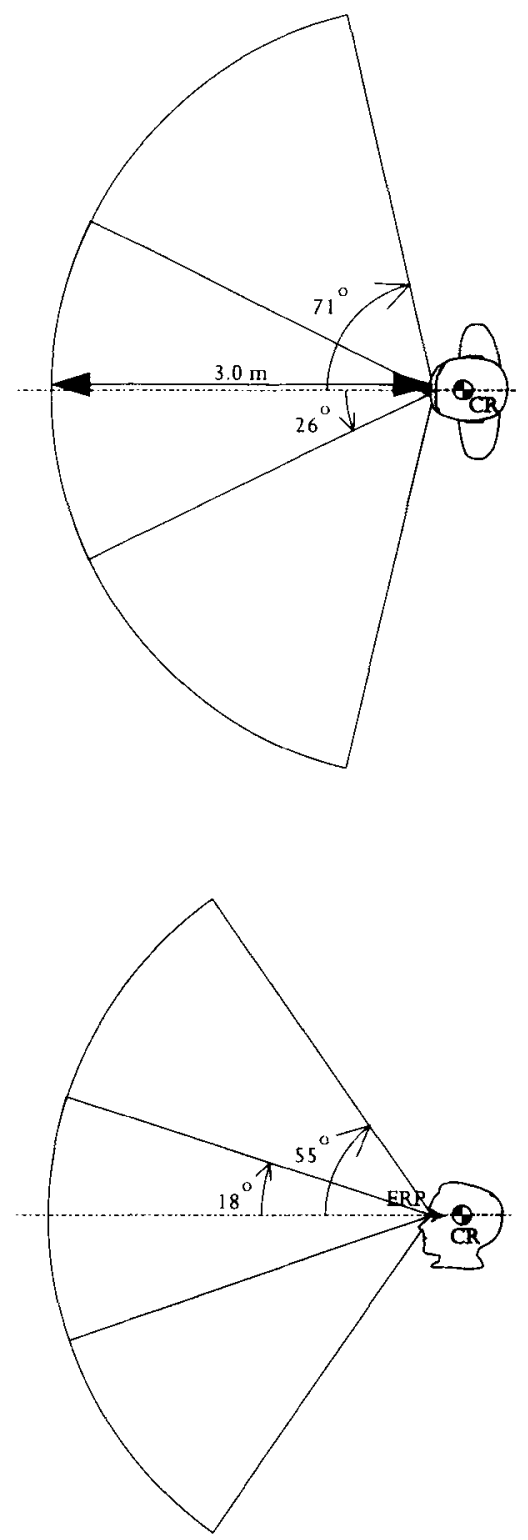

Figure 2. Top and side views of the background $(142 \times 110)$ and inset $(52 \times 36)$ visual angles. $C R$, the center of the subject's head, which was the rotation center of the roll and the yaw motions tested; ERP, the eye reference point in between the subject's eyes. Reprinted from Journal of Vestibular Research, 8, F. A. М. van der Steen, An earth-stationary perceived visual scene during roll and yaw motions in a flight simulator, 411-425, Copyright 1998, with permission from Elsevier Science.

the subject for surge. This visual velocity was kept constant throughout the entire experimental session, independent of subsequent inertial motions.

\section{Inertial Motion}

No inertial stimulus was applied for $5 \mathrm{sec}$ from the start of visual scene motion. Next, a series of trials was provided at regular intervals of $5 \mathrm{sec}$. These relatively brief intervals were chosen to avoid vection adaptation (see Berthoz \& Droulez, 1982, for an overview). Each trial consisted of a $3-\mathrm{sec}$ presentation of an inertial stimulus in smoothed acceleration steps, returning the cockpit to the initial mid- position (Figure 6), followed by a 2 -sec absence of inertial motion. The inertial motion direction in one experimental session was roll, yaw, surge, or sway. No motions were applied in other directions.

The inertial translations were constrained to the maximum simulator base excursion of $1.1 \mathrm{~m}$ for both the surge and sway motions. The inertial rotations were constrained to a maximum roll angle of $30 \mathrm{deg}$ and a maximum yaw angle of $40 \mathrm{deg}$.

\section{Task}

The subject was instructed to fixate objects in the central part of the high-resolution inset. The subject was instructed to avoid head and limb movements as much as possible during the experimental sessions. Furthermore, the subject was instructed to verbally report perceived self-motion and perceived visual scene motion throughout the experimental sessions.

In the first part of each experimental session, following the onset of visual scene motion, the subject had to indicate the instant at which the visual scene was perceived as earth stationary by briefly pulling the side-stick. In the second part of the session, the subject perceived an earth-stationary visual scene (saturated vection). The subject indicated each time when the visual scene was not perceived as earth stationary anymore, due to an inertial motion profile, by pushing a button on the side-stick.

\section{Staircase Procedure}

The amplitudes of the inertial motions were varied over trials using a staircase procedure. This procedure was applied to determine the smallest inertial amplitudes at which the perception of an earth-stationary visual scene was lost and, hence, saturated vection was impaired. These inertial amplitudes are referred to as the inertial threshold amplitudes.

The acceleration amplitude of the first inertial stimulus in the staircase was $0.57 \mathrm{deg} / \mathrm{sec}^{2}$ (which is equal to $0.01 \mathrm{rad} / \mathrm{sec}^{2}$ ) for roll and yaw motions, and $0.05 \mathrm{~m} / \mathrm{sec}^{2}$ for surge and sway motions. These inertial motion profiles were assumed to be below inertial self-motion perception threshold for each motion direction (Berthoz \& Droulez, 1982; Guedry, 1974; Hosman \& van der Vaart, 1978; Peters, 1969), although thresholds for this specific kind of inertial motion have not been documented to date. The initial amplitude of the staircase step size was equal to the amplitude of the first inertial stimulus, multiplied by a random factor between 0.8 and 1.2 to avoid identical staircases. Until the button was pressed. indicating that the subject no longer perceived the visual scene to be earth stationary, the inertial amplitude was continuously incremented by the step size amplitude and presented in the next trial. Then, the step size amplitude was halved. Until the button press was absent in a trial, the inertial amplitude was continuously decremented by the step size amplitude and presented in the next trial. Then, the step size was halved again. Until a button was pressed, the inertial amplitude was continuously incremented by the step size amplitude and presented in the next trial. This procedure of halving the step size and incrementing and decrementing the inertial amplitude continued until the step size became less than one eighth of the initial step size. Then, the staircase procedure was complete and the session was stopped. The session was also stopped when the number of trials exceeded 30 in order to avoid fatigue in the subject. A short break of about 1 min was taken between each session.

Experimental sessions for each inertial motion direction were performed three times. Additional sessions were conducted. however, when the total amount of trials from the staircase procedure was smaller than 30 .

\section{Registration}

The time histories of visual scene position. inertial acceleration, stick deflection, and button presses were recorded. The subject's verbal comments about perceived self-motion and visual scene motion were reported by the experimenter. The subject was asked whether he was feeling well at regular intervals in order to determine whether 


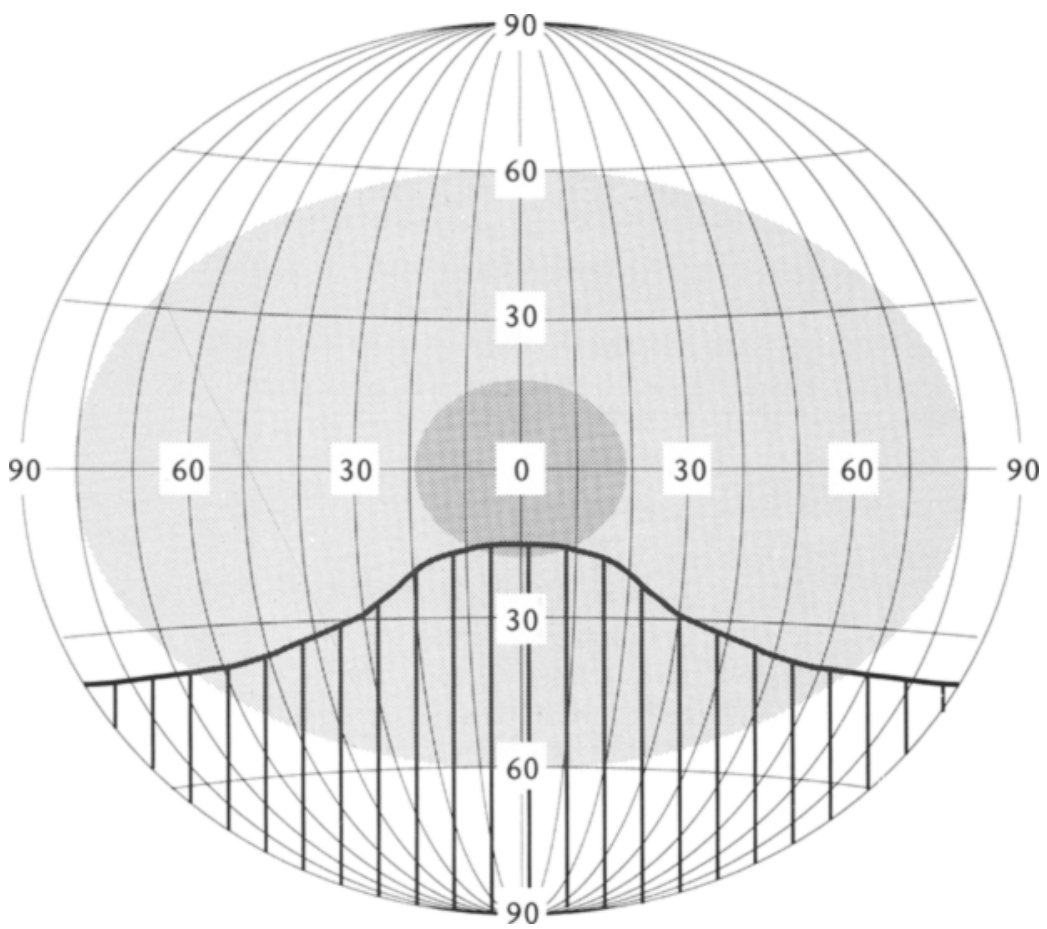

Figure 3. Visual scene geometry, depicting the high-resolution inset (shaded heavily), the background (shaded lightly), and the lower midpart, which was occluded by the cockpit (hatched vertically). The numbers indicate the angles (in degrees) from the horizontal line connecting the ERP and the visual scene center. Reprinted from Journal of Vestibular Research, 8, F. A. M. van der Steen, An earth-stationary perceived visual scene during roll and yaw motions in a flight simulator, 411-425, Copyright 1998, with permission from Elsevier Science.

he was experiencing motion sickness, as sometimes happens during these kinds of experiments (Hu, Stern, Vasey, \& Koch, 1989; Tiande \& Jingshen, 1991).

\section{Data Reduction}

The instant the subject pulled the side-stick, indicating perception of an earth-stationary visual scene, was recorded and is referred to as time to stick pull. The mean time to stick pull of each subject was determined by averaging the times registered in each session.

The responses "button press" and "no button press" from each trial were assigned probability values of 1 and 0 , respectively. The responses were grouped for each separate inertial motion direction tested. A psychometric curve from a normal distribution was fitted to the responses using a least squares criterion. The inertial thresh- old amplitudes at which the visual scene was perceived as not earth stationary were determined at the amplitude that resulted in a button press probability of .5 .

When appropriate, the standard deviation of results was calculated. Significant differences among subjects in time to stick pull and in inertial threshold amplitudes were determined using analyses of variance (ANOVAs) with a $p<.01$ criterion.

\section{RESULTS \\ Vection Induction}

Time histories from an experimental session of 1 subject are depicted in Figure 7. This figure shows the registered stimuli (visual and inertial) and the subject's responses (stick pull and button presses).

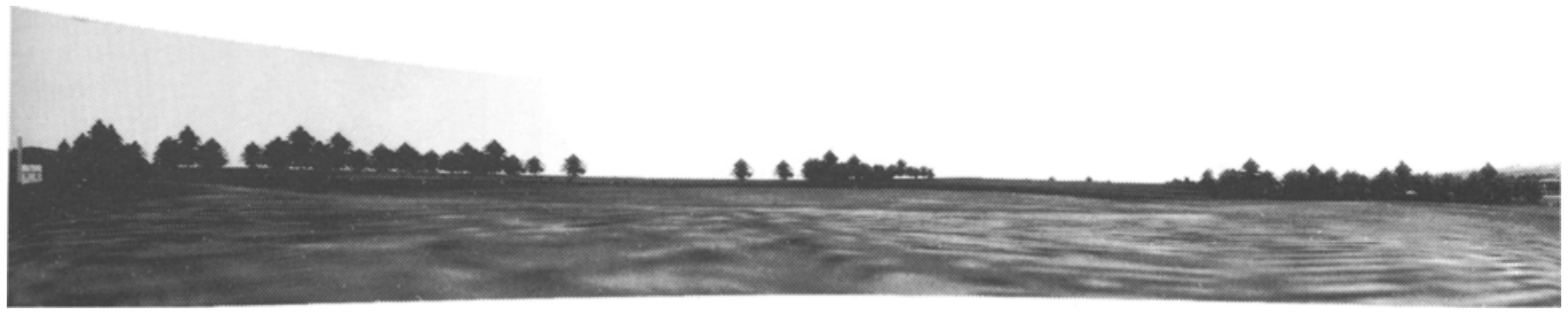

Figure 4. Part of the visual scene (covering about 180 deg horizontally) used in the yaw vection experiment. 

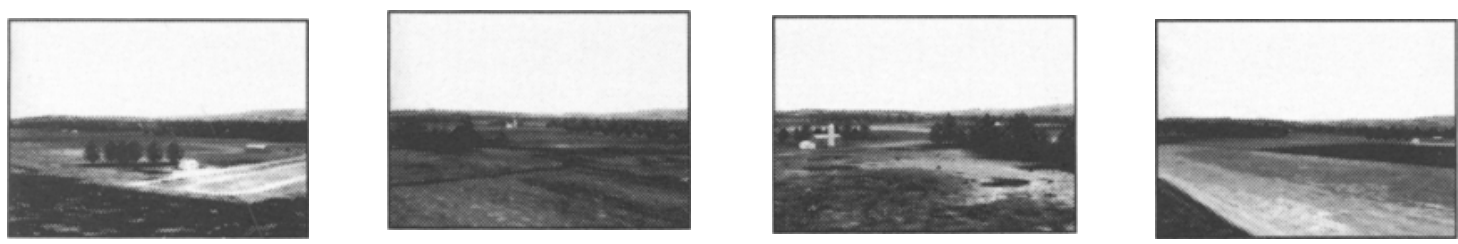

Figure 5. Subsequent parts (from left to right) of the visual scene used in the surge vection experiment.

None of the subjects showed or reported symptoms of motion sickness.

\section{Verbal reports}

None of the subjects reported a perception of selfacceleration between the time of visual motion onset and the time to saturated vection and, no subject reported perceiving that the visual scene decelerated during this period of time. Instead, the subjects regularly reported an indeterminate phase during this period of time between, first, the perception of a moving visual scene and no self-motion and, next, the perception of self-motion in an earth-stationary visual scene.

\section{Yaw vection}

The times to stick pull are depicted in Figure 8. Most subjects occasionally forgot to pull the stick while hav- ing verbally reported experiencing an earth-stationary visual scene. Subject 5 pulled the stick only once; his data were excluded from group analysis. The mean group time to stick pull was $3.0 \mathrm{sec}(S D=2.5 \mathrm{sec})$. The rather large $2.5-\mathrm{sec} S D$ was mainly due to Subject 1's large time to stick pull of $6.7 \mathrm{sec}(S D=2.0 \mathrm{sec})$, which was significantly longer than those of the rest of the group. During this subject's sessions, occasionally the first (subliminal) inertial stimulus already had been presented before the stick was pulled. Figure 8 also shows that Subject 4 experienced saturated vection almost immediately.

\section{Surge vection}

The mean group time to stick pull was $2.7 \mathrm{sec}(S D=$ $0.9 \mathrm{sec}$; Figure 8). No subject showed significantly shorter or longer times relative to those of the group.
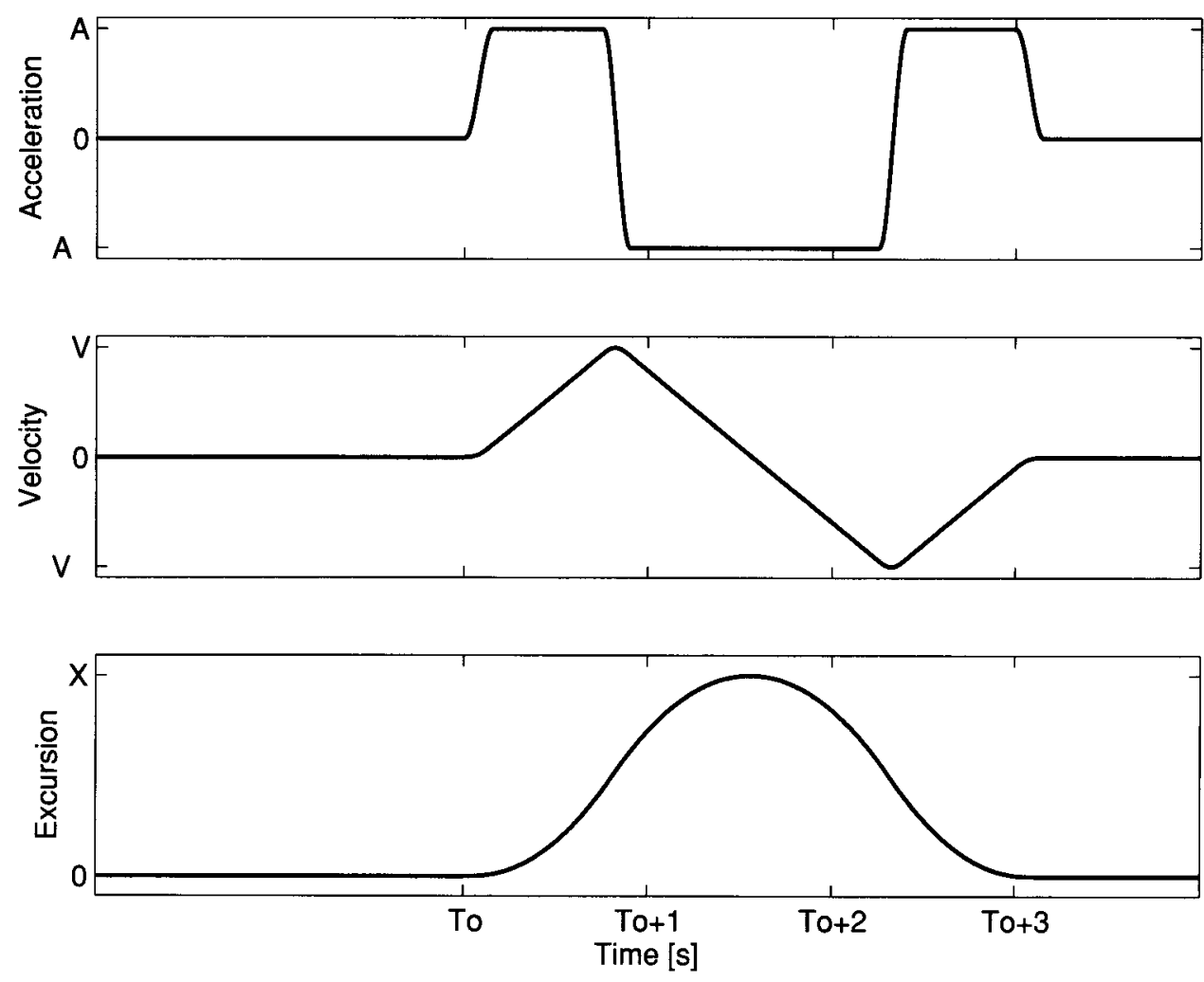

Figure 6. The stimulus motion profile, showing the acceleration (top), velocity (middle), and excursion (bottom). Following the stick deflection at the time $t=T_{0}$, a constant acceleration $\mathrm{A}$ was provided for $0.75 \mathrm{sec}$, followed by a deceleration $-A$ for $1.50 \mathrm{sec}$ and an acceleration $A$ for $0.75 \mathrm{sec}$. 

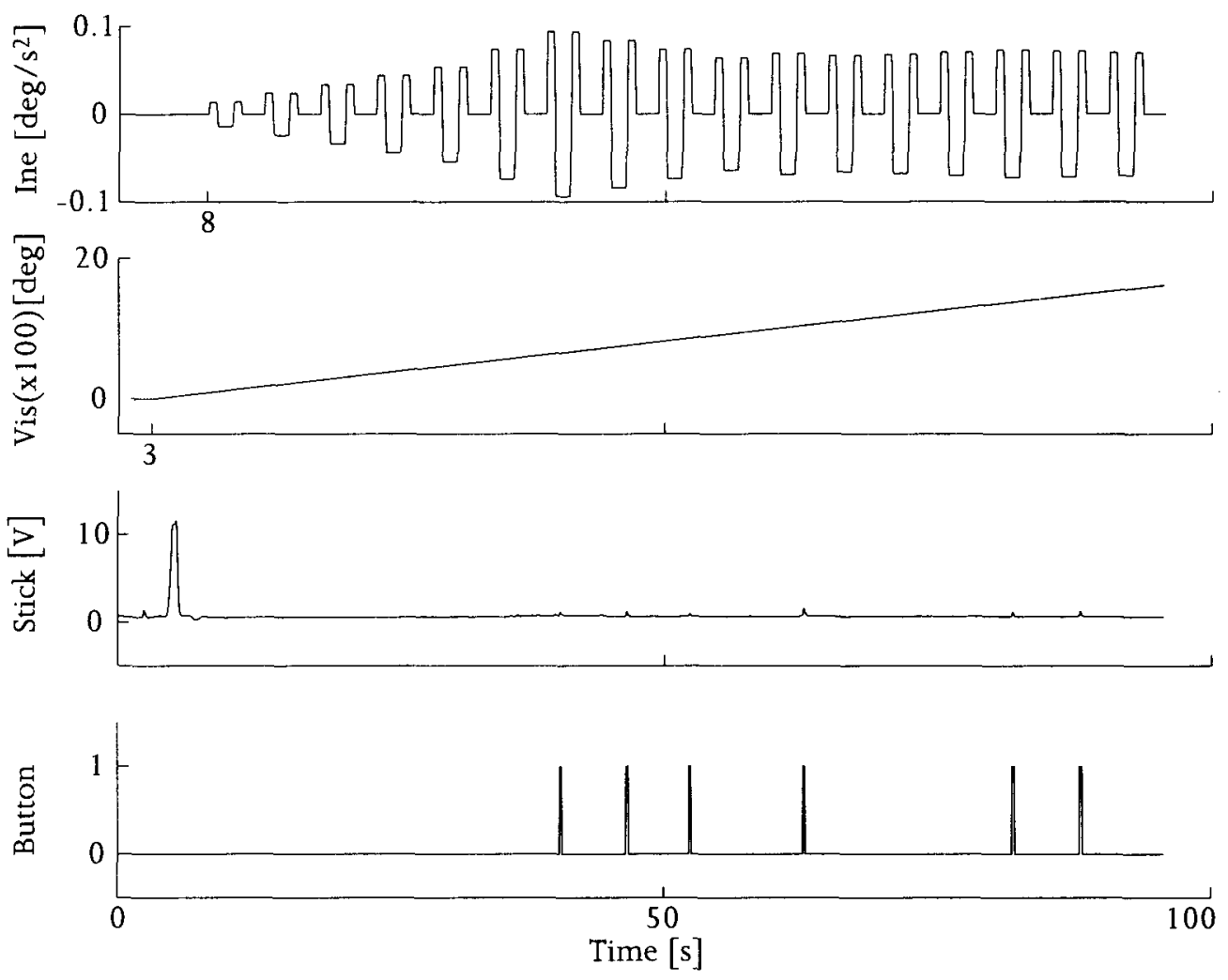

Figure 7. Time histories from an experimental session of 1 subject. From top to bottom: inertial stimulus acceleration, visual stimulus excursion, stick deflection, and button presses.

\section{Vection Impairment}

\section{Verbal reports}

The verbal reports during the experimental sessions were similar for all subjects in both the yaw and surge experiments. During the first few trials, in which inertial motion was applied, the subjects did not perceive any selfmotion except from saturated vection. After a few trials, however, the subjects reported perceiving self-acceleration without being able to state the direction. This occurred at about $2-3 \mathrm{deg} / \mathrm{sec}^{2}$ for intertial yaw and roll motions, and at about $0.10-0.15 \mathrm{~m} / \mathrm{sec}^{2}$ for sway and surge. Meanwhile, the visual scene was perceived as remaining earth stationary. A few trials later, the subjects perceived the inertial motion direction correctly but the visual scene was still perceived as earth stationary. During the inertial roll and yaw motions, the perception of an earth-stationary vi-
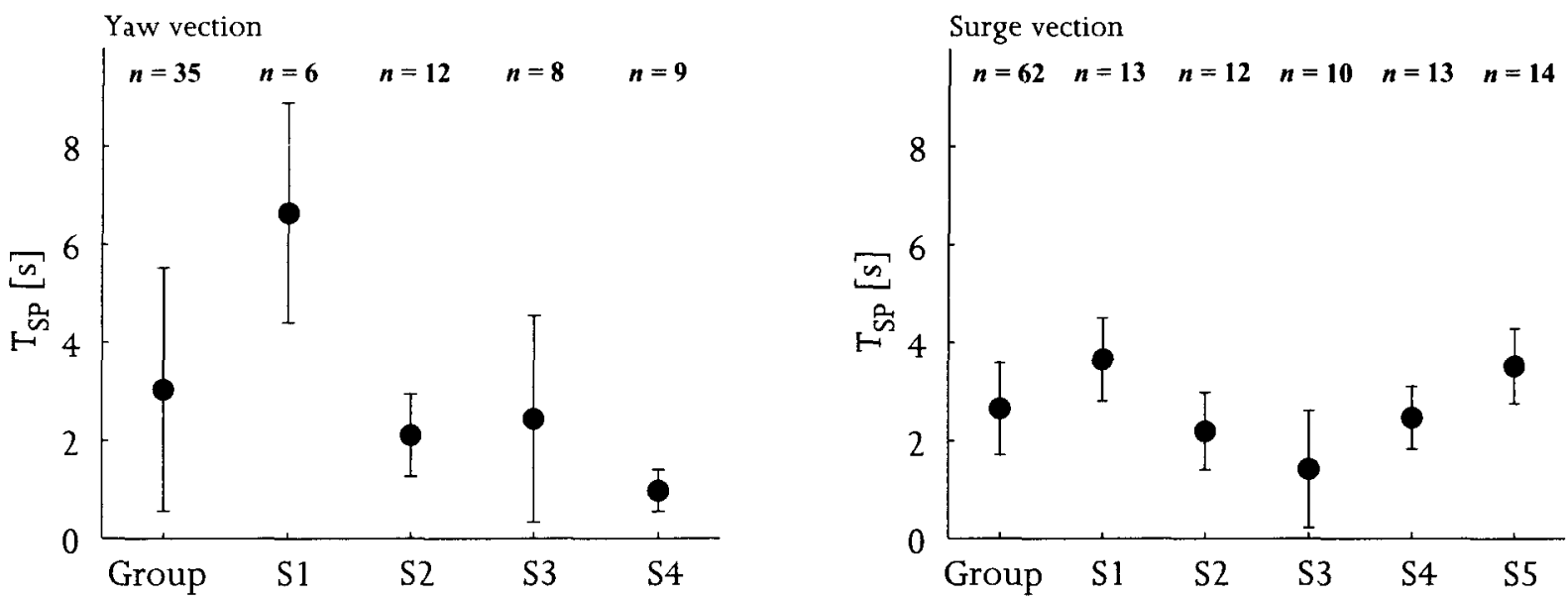

Figure 8. The times to stick pull ( $\left.T_{\mathrm{SP}}\right)$ from both the yaw vection and surge vection experiments, showing the mean and standard deviations of the individual subjects and the group. The number of sessions from which these results were obtained is indicated by $n$. 


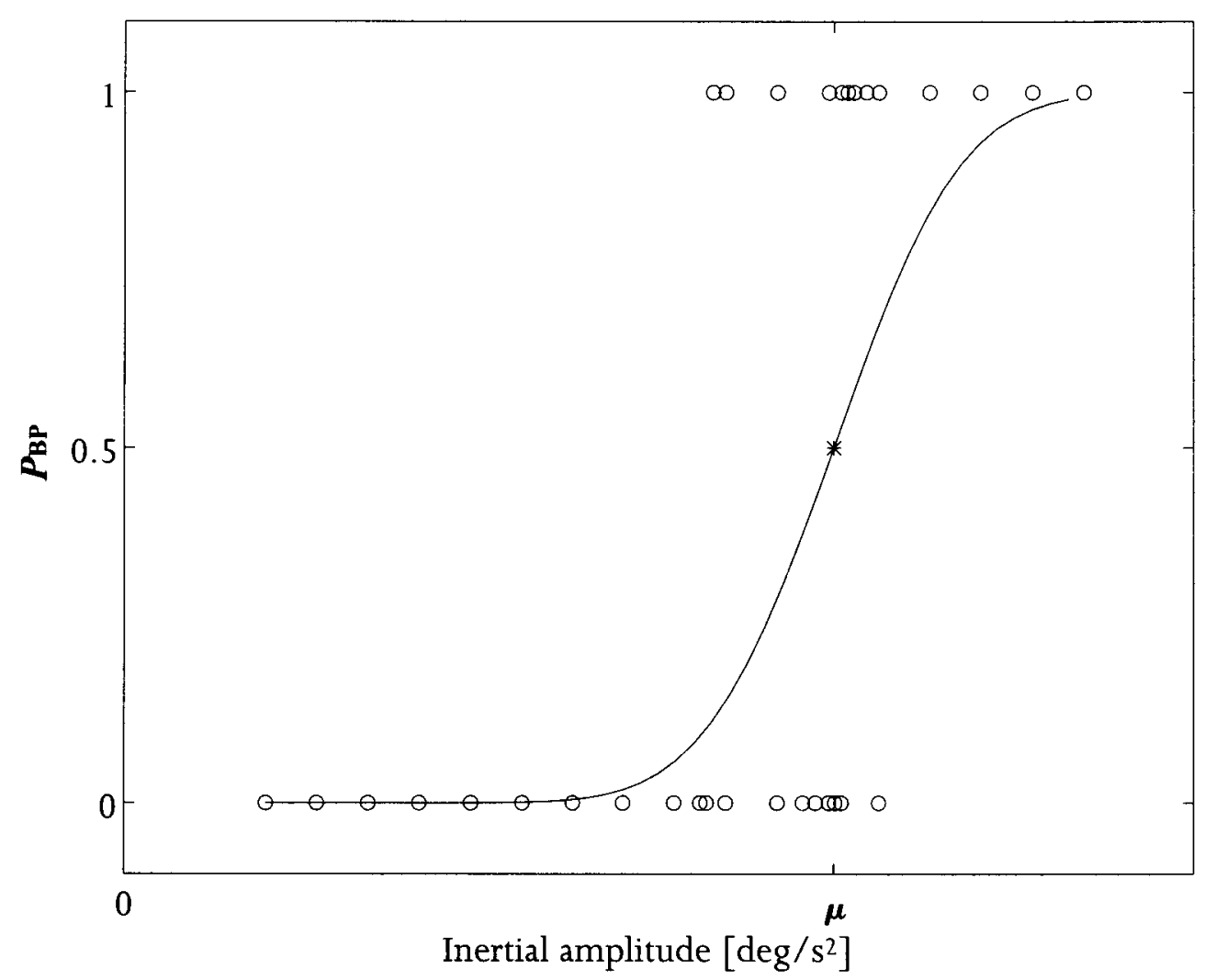

Figure 9. One subject's buttonpress responses from one inertial motion direction tested as a function of inertial stimulus amplitude. The responses were assigned a value of 1 when the button was pressed and a value of 0 when a button press was absent within each trial. The psychometric curve from a normal distribution function was fitted using a least squares criterion. The inertial threshold amplitude was determined at a .5 button press probability $\left(P_{\mathrm{BP}}\right)$ and is indicated by $\mu$.

sual scene was lost at amplitudes slightly above the inertial amplitudes at which the inertial motion direction was perceived correctly. Inertial surge and sway motions, on the other hand, did not affect the perception of an earthstationary visual scene until the amplitude had become rather large: see the following paragraphs on yaw vection and surge vection. When saturated vection was impaired, as evidenced by the perception of a moving visual scene with respect to an earth-fixed reference frame, the subjects reported visual scene motion that was similar to perceived self-motion from the applied inertial motion. When the inertial motion was a roll motion, for example, the subjects reported both a roll self-motion and a roll motion of the visual scene in the same direction. When inertial motion was absent, during the last 2 sec of each trial, the subjects reported perceiving the visual scene to be earth stationary again almost immediately.

\section{Yaw vection}

An example of I subject's button press responses from one inertial motion direction tested and the psychometric curve fit to these responses is depicted in Figure 9. Figure 10 shows the individual and group threshold amplitudes at which the visual scene was no longer perceived as earth stationary for each of the four inertial motion di- rections tested. Subject 5 had significantly higher thresholds for roll and yaw relative to the group mean. Subject 1 had significantly higher thresholds for sway. Comparison of the thresholds from rotations (yaw and roll) and translations (surge and sway) with their respective (estimated) inertial self-motion perception thresholds indicates that the thresholds for rotations were quite low, whereas the thresholds for translations were rather large (Figure 10).

\section{Surge vection}

Subject 5 had, as was found in the yaw vection experiment, significantly higher thresholds for inertial roll and yaw motions relative to those of the group. Subject 4 had significantly higher thresholds for surge, and Subject 1 had significantly lower thresholds for sway motion. Again, as in the yaw vection experiments, the thresholds for rotations were quite low, whereas the thresholds for translations were rather large (Figure 10).

\section{DISCUSSION}

\section{Induction of Saturated Vection}

During the yaw vection experiment, Subject 1's times to stick pull occasionally occurred later than the presentation of the first inertial profile. Although vection yet 

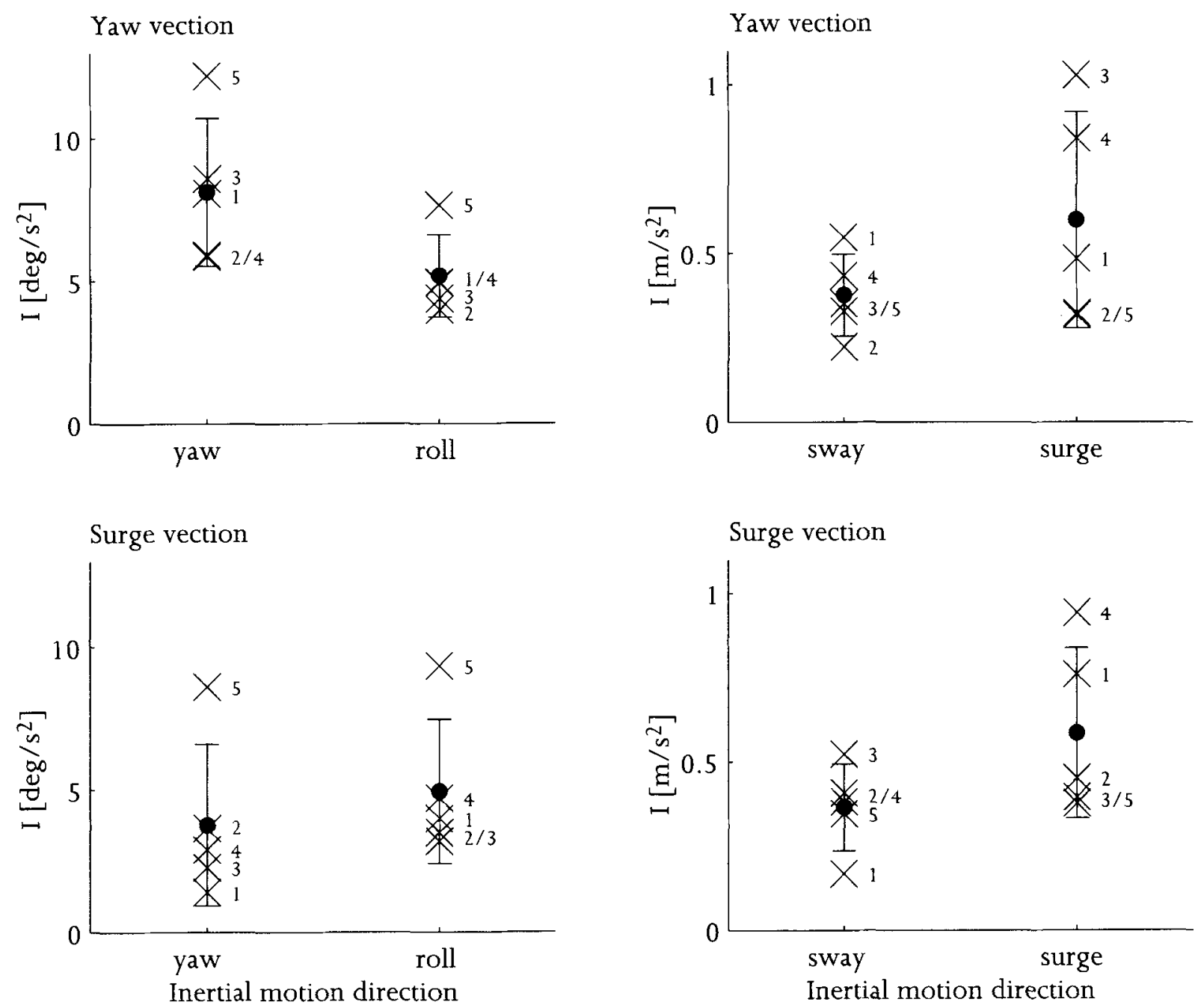

Figure 10. The inertial threshold amplitudes at which vection was impaired for the different inertial motion directions tested: yaw, roll, sway, and surge. The individual thresholds are indicated by crosses with corresponding subject numbers at the right. The group means and standard deviations are indicated by solid circles and error bars, respectively.

had to saturate while inertial motion was provided, it is unlikely that the induction of saturated vection was influenced by this inertial stimulus because of the subliminal inertial amplitude of the first inertial stimulus for each motion direction tested.

It should be noted that the time to stick pull, indicating saturation of vection, consists of the actual time to saturated vection and a reaction time. Even with a reaction time included, the mean group time to saturated yaw vection was substantially slower than the times found in optokinetic drum experiments in which the same visual velocity profile and amplitude was tested. Brandt et al. (1973), for example, reported a mean group saturation time of about $10 \mathrm{sec}$ for visual drum velocities between 10 and $30 \mathrm{deg} / \mathrm{sec}$. Howard and Howard (1994) reported even longer saturation times of $20-30 \mathrm{sec}$. Also, the mean group time to saturated surge vection was substantially slower than the times found in similar experiments (e.g., Berthoz \& Droulez, 1982). Because the only salient dif- ference between other experiments and the present experiments is the visual scene, it must be concluded that the visual scene used here was more optokinetic than the scenes used in the other experiments.

No self-acceleration was perceived between onset of visual scene motion and saturated vection. This indicates that the visual scene was unable to arouse a perception of self-acceleration during the applied steps of visual scene velocity but rather aroused a sudden change in the amplitude of perceived self-velocity. This contrasts the findings from similar experiments using optokinetic drums (Brandt et al., 1973; Dichgans \& Brandt, 1978), in which subjects reported a gradual buildup of self-velocity and a gradual decrease of visual scene velocity.

\section{Impairment of Saturated Vection}

The results show that both saturated yaw and surge vection are more robust for the translatory than for the rotational inertial motions since the rotational inertial 
threshold amplitudes were much closer to the amplitudes at which the applied inertial motion was perceived than the translational inertial threshold amplitudes. This is most likely to be attributed to the absence of additional optic flow during both the yaw and the roll inertial motions, which, if present, would have been quite persuasive: Yaw and roll motions result in a rotation of the whole visual field (Koenderink, 1990). The absence of additional optic flow from inertial surge and sway motions, on the other hand, was relatively insignificant for the scene tested. Since the visual objects were at a relatively large distance from the subject (Figures 4 and 5)-the maximum translatory surge or sway excursion of about $1 \mathrm{~m}-$ the maximum simulator base excursion was quite small. When the visual objects are closer, it is expected that the inertial threshold amplitudes will be smaller. Then, the lack of additional translatory motion of the visual scene will lead to perceived motion of the visual scene at smaller inertial amplitudes.

\section{GENERAL DISCUSSION}

None of the subjects suffered from motion sickness, although they were exposed to vection and to incongruous combinations of inertial and visual motion stimulations that could induce motion sickness (Hu et al., 1989; Kolasinski, 1995; Oman, 1990; Tiande \& Jingshen, 1991; Yardley, 1992). A relatively small number of subjects, however, were tested here, none of whom had a history of extraordinary motion sickness susceptibility. Furthermore, the inertial stimulations were brief, which might reduce the chance of motion sickness (see Kolasinski, 1995 , for an overview).

Saturation times and thresholds are likely to depend on several task- and stimulus-related parameters. In the present experiments, the subject's attention was focused on perceiving a moving visual scene and acceleration of the body. When the subject performs an additional control task or some other distracting task, for example, the saturation time should be expected to be lower and the inertial threshold amplitudes to be larger. It is therefore argued that, for the motion stimuli tested and the setup used, the saturation times are largest and the inertial amplitudes are lowest.

In contrast to the findings from, for example, optokinetic drum experiments (Brandt et al., 1973; Dichgans \& Brandt, 1978), no gradual buildup of self-velocity and no concurrent gradual decrease of visual scene velocity were found. Furthermore, the times to saturation were much earlier than those found in similar vection experiments described in the literature. This must be ascribed to the different visual stimulus used in the present experiments. Also, the subjects perceived a rather sudden change in self-velocity and visual scene velocity with respect to an earth-fixed reference frame. Such a sudden change can also be seen in the train illusion that was mentioned in the introduction of this article. When the train passenger experiences vection and looks out the opposite window at the station platform, the perception of self-motion quickly vanishes without any perception of self-acceleration. Hence, a quick perceptual switch occurs from an adjacent train-fixed reference frame to a station-fixed frame of reference to which perceived self-motion is related.

Whereas findings from optokinetic drum experiments suggest that the visual stimulus solid angle is the key parameter in the dynamics of saturated vection induction, the present results indicate that it is more likely that the scene layout is important-what is out there in the scene, as indicated by Wolpert (1990), rather than how many. This is in contradiction with the hypothesis of Howard and Howard (1994), who stated that the dynamics of vection induction is a matter of retinal location. It may tentatively be concluded from the present results that saturated vection is induced more easily if the visual scene represents a natural environment, supporting Wolpert's statement. A possible explanation is that a natural scene is less sensitive to an ambiguous perception of either motion of the environmental scene or motion of a visual pattern or object, which, for example, can occur during the perception of optokinetic drum motion in the laboratory. It is likely that the optokinetic drum can more easily be perceived as an intermediate scene - an object - between the subject and the natural environment. One of the main reasons for that is that the optokinetic drum is at a relatively small distance of about $1 \mathrm{~m}$ and does not represent a more distant scene. The present scene, on the other hand, was physically at a greater distance $(3 \mathrm{~m})$ and thus represented an environment at a great distance, including a variety of depth cues.

The perception of an earth-stationary visual scene seems to be a good measure of the realism of simulated self-motion (van der Steen, 1998). As a result, the inertial threshold amplitudes that were found in the present experiments on impairment of saturated vection determine the largest amount of inertial motion that may be applied during saturated vection while self-motion is still perceived as realistic. It should be noted, however, that these thresholds are not generally applicable to all stimulus conditions. The thresholds found here apply only to the specific visual and inertial stimuli tested, but can serve as a reference for similar setups and motion stimuli. It should furthermore be noted that substantial interindividual differences existed among the inertial thresholds. This can be seen from the significantly different thresholds for 3 of the 5 subjects for one or more inertial motions (Figure 10). Only Subjects 2 and 3 had inertial threshold amplitudes that did not differ significantly from those of the group for all conditions tested.

The subjects' verbal reports show that no selfacceleration was perceived between two different amplitudes of perceived self-velocity. It can thus be concluded that there is no single and continuous self-motion signal from which perceived self-velocity and perceived selfacceleration result. This is an important contribution to the design of self-motion perception theories (Mergner, Rumberger, \& Becker, 1996; Wertheim, 1994). The present 
results suggest that self-acceleration is mainly perceived through stimulation of the inertial sensory systems. Visual scene velocity with respect to the observer induces mainly a perception of self-velocity, not self-acceleration.

\section{CONCLUSIONS}

Saturated vection is induced more rapidly when the visual scene represents a "natural environment" than when the scene is more "artificial," such as a vertically striped drum or a random dot pattern. During the induction of saturated vection, the subjects did not perceive any selfacceleration or visual scene deceleration but rather perceived a sudden change in self-velocity and visual scene velocity with respect to an earth-fixed reference frame. Saturated vection is more robust for translations than for rotations.

\section{REFERENCES}

ANDERSEN, G. J., \& BraunsteIn, M. L. (1985). Induced self-motion in central vision. Journal of Experimental Psychology: Human Perception \& Performance, 11, 122-132.

BerThoZ, A., \& Droulez, J. (1982). Linear self-motion perception. In A. H. Wertheim, W. A. Wagenaar, \& H. W. Leibowitz (Eds.), Tutorials on motion perception (pp. 157-198). New York: Plenum.

Berthoz, A., Pavard, B., \& Young, L. R. (1975). Perception of linear horizontal self-motion induced by peripheral vision (linear vection): Basic characteristics and visual-vestibular interactions. Experimental Brain Research, 23, 471-489.

BLES, W. (1981). Stepping around: Circular vection and Coriolis effects. In J. [B.] Long \& A. [D.] Baddeley (Eds.), Attention and performance IX (pp. 47-61). Hillsdale, NJ: Erlbaum.

Brandt, T., BÜCHElE, W. \& ARNold, F. (1977). Arthrokinetic nystagmus and ego-motion sensation. Experimental Brain Research, 30 , 331-338.

Brandt, T., Dichgans, J., \& Koenig, E. (1973). Differential effects of central versus peripheral vision on egocentric and exocentric motion perception. Experimental Brain Research, 16, 476-491.

BRandT, T., WIST, E., \& Dichgans, J. (1971). Optisch induzierte Pseudocoriolis Effekte und Circularvektion [Optically induced pseudo-coriolis effects and circular vection]. Archiv für Psychiatrie und Nervenkrankheiten, 214, 365-389.

BütTner, U., \& HenN, V. (1981). Circularvection: Psychophysics and single-unit recordings in the alert monkey. Annals of the New York Academy of Sciences, Vol. 374, pp. 274-283. New York: New York Academy of Sciences.

Carpenter-Smith, T. R., Futamura, R. G., \& Parker, D. E. (1995). Inertial acceleration as a measure of linear vection: An alternative to magnitude estimation. Perception \& Psychophysics, 57, 35-42.

Dichgans, J., \& BRANDT, T. (1978). Visual-vestibular interaction: Effects on self-motion perception and postural control. In R. Held, H. W. Leibowitz, \& H. L. Teuber (Eds.), Handbook of sensory physiology: Vol. 8. Perception (pp. 756-804). New York: Springer-Verlag.

Fischer, M. H., \& KoRnMülLER, A. E. (1930). Optokinetischer ausgelöste Bewegungswahrnemung und optokinetischer Nystagmus [Optically induced motion perception and optokinetic nystagmus]. Journal für Psychologie \& Neurologie, 41, 273-308.

Guedry, F. E. (1974). Psychophysics of vestibular sensation. In H. H. Kornhuber (Ed.), Handbook of sensory physiology (Vol. 6, pp. 1154). Berlin: Springer-Verlag.

Hosman, R. J. A. W., \& VAN der VAaRT, J. C. (1978). Vestibular models and thresholds of motion perception. Results of tests in a flight simulator (Report No. LR-265). Delft: Delft University of Technology, Faculty of Aerospace Engineering, Delft, the Netherlands.

HoWARD, I. P. (1982). Human visual orientation. Chichester, U.K.: Wiley. HowARD, I. P. (1986). The perception of posture, self-motion, and the visual vertical. In K. R. Boff, L. Kaufman, \& J. P. Thomas (Eds.), Handbook of perception and human performance: Vol. I. Sensory processes and perception (pp. 18.1-18.62). New York: Wiley.

HOWARD, I. P., \& HowaRd, A. (1994). Vection: The contributions of absolute and relative visual motion. Perception, 23, 745-751.

Hu, S., Stern, R. M., Vasey, M. W., \& Кoch, K. L. (1989). Motion sickness and gastric myolectric activity as a function of speed of rotation of a circular vection drum. Aviation, Space, \& Environmental Medicine, 60, 411-414.

KoenderinK, J. J. (1990). Some theoretical aspects of optic flow. In R. Warren \& A. H. Wertheim (Eds.), Perception and control of selfmotion (pp. 53-68). Hillsdale, NJ: Erlbaum.

KOLASINSKI, E. M. (1995). Simulator sickness in virtual environments (Technical Rep. No. 1027). U.S. Army.

LACKNER, J. R. (1977). Induction of illusory self-rotation and nystagmus by a rotating sound-field. Aviation, Space, \& Environmental Medicine, 48, 129-131.

LACKNER, J. R., \& DiZio, P. (1984). Some efferent and somatosensory influences on body orientation and oculomotor control. In L. Spillmann \& B. R. Wooten (Eds.), Sensory experience, adaptation, and perception. Hillsdale, NJ: Erlbaum.

МАCH, E. (1967). Grundlinien der Lehre von den Bewegungsempfindungen. Amsterdam: Bonset. (Original work published 1875)

Mergner, T., Rumberger, A., \& BeCKer, W. (1996). Is perceived angular displacement the time integral of perceived angular velocity? Brain Research Bulletin, 40, 467-471.

OMAN, C. M. (1990). Motion sickness: A synthesis and evaluation of the sensory conflict theory. Canadian Journal of Physiology \& Pharmacology, 68, 294-303.

Peters, R. A. (1969). Dynamics of the vestibular system and their relation to motion perception, spatial disorientation and illusions (Report CR-1309). Washington, DC: NASA.

TIANDE, Y., \& JingSHEN, P. (1991). Motion sickness severity under interaction of vection and head movements. Aviation, Space, \& Environmental Medicine, 62, 141-144.

VAN DER STEEN, F. A. M. (1998). An earth-stationary perceived visual scene during roll and yaw motions in a flight simulator. Journal of Vestibular Research, 8, 411-425.

WertheIM, A. H. (1994). Motion perception during self-motion: The direct versus inferential controversy revisited. Behavioral \& Brain Sciences, 17, 293-355.

WolPERT, L. (1990). Field-of-view information for self-motion perception. In R. Warren \& A. H. Wertheim (Eds.), Perception and control of self-motion (pp. 101-126). Hillsdale, NJ: Erlbaum.

YARDLEY, L. (1992). Motion sickness and perception: A reappraisal of the sensory conflict approach. British Journal of Psychology, 83, 449-471.

\section{NOTES}

1. This ease stands in contrast to the difficulty of determining the amplitude of perceived self-motion during unsaturated vection (see Carpenter-Smith, Futamura, \& Parker, 1995, for a brief review).

2. OL and ftL are terms that are commonly used to express a simulator visual display's resolution and luminance, respectively. OLP is a metric for the resolution since it requires 1 pixel on and 1 pixel off to be the minimum resolvable element in a visual display. $\mathrm{A} \mathrm{ftL}$ is a measure of luminance and corresponds to an emission of 1 lumen per square foot from a perfectly diffuse radiator.

(Manuscript received December 31, 1997; revision accepted for publication August 19, 1998.) 